\title{
SUR LE DÉVELOPPEMENT EN FRACTION CONTINUE D'UNE GÉNÉRALISATION DE LA CUBIQUE DE BAUM ET SWEET
}

\author{
ALINA FIRICEL
}

\section{INTRODUCTION}

Il y a une trentaine d'années, les travaux de Baum et Sweet [1] ont ouvert un nouveau domaine de recherche sur l'approximation diophantienne dans les corps de séries formelles à coefficients dans un corps fini, par le biais du développement en fraction continue. Ces auteurs ont notamment donné l'exemple d'une série formelle à coefficients dans le corps fini $\mathbb{F}_{2}$, algébrique de degré 3 sur $\mathbb{F}_{2}(T)$, ayant un développement en fraction continue avec des quotients partiels qui sont tous des polynômes en $T$ de degré 1 ou 2 . Dix ans plus tard, Mills et Robbins [5] ont décrit un algorithme qui leur a permis de donner le développement explicite en fraction continue pour la série formelle cubique de Baum et Sweet. Ces travaux ont mis en lumière un sous ensemble de séries formelles algébriques, obtenues comme points fixes de la composée d'une homographie à coefficients entiers (polynômes) avec le morphisme de Frobenius; ces séries sont alors appelées hyperquadratiques. Le développement en fraction continue a pu être donné explicitement (voir [2, 5, 6] pour plus de références) pour de nombreux exemples de ces séries formelles.

Nous avons observé que pour tout nombre premier $p$ et $r=p^{t}$, l'équation

$$
T X^{r+1}+X-T=0
$$

a une unique solution dans le corps $\mathbb{F}_{p}\left(\left(T^{-1}\right)\right)$. Pour $r=p=2$ cette solution est la cubique de Baum et Sweet. Dans cet article, nous donnons le développement en fraction continue de cette solution pour $r>2$ (voir la partie 3). On peut par ailleurs remarquer que si l'on remplace $r$ par 2 dans les formules obtenues, alors le développement obtenu est impropre (une sous-suite de quotients partiels tend vers 0 dans $\left.\mathbb{F}_{p}\left(\left(T^{-1}\right)\right)\right)$. Cependant, cette expression, lorsqu'elle est tronquée et rendue propre, donne le développement qui a été obtenu dans [5].

Pour obtenir ce développement nous utilisons une méthode déjà utilisée par Lasjaunias dans [3], qui, bien que proche de l'algorithme de Mills et Robbins, en diffère un peu. Pour illustrer cette méthode, nous l'appliquons dans un premier temps à un autre exemple de série formelle hyperquadratique, à coefficients dans $\mathbb{F}_{p}$. Cet exemple, très célèbre, a été introduit par Mahler [4] dans un article fondateur sur l'approximation diophantienne dans les corps de fonctions.

Nous rappelons brièvement les notations utilisées. Dans ce texte, $p$ est un nombre premier, $\mathbb{F}_{p}$ désigne le corps fini à $p$ éléments, $\mathbb{F}_{p}[T], \mathbb{F}_{p}(T)$ et $\mathbb{F}_{p}\left(\left(T^{-1}\right)\right)$ sont, respectivement, l'anneau des polynômes, le corps des fonctions rationnelles et le corps des séries formelles (en $1 / T$ ) de la variable $T$ sur $\mathbb{F}_{p}$. Ainsi

$$
\mathbb{F}_{p}\left(\left(T^{-1}\right)\right)=\{0\} \cup\left\{\sum_{k \leq k_{0}} u_{k} T^{k}, k_{0} \in \mathbb{Z}, u_{k} \in \mathbb{F}_{p}, u_{k_{0}} \neq 0\right\} .
$$

Ce corps de séries formelles est muni d'une valeur absolue ultramétrique définie par $|\alpha|=|T|^{k_{0}}$ et $|0|=0$, où $|T|$ est un réel fixé strictement supérieur à 1 . De plus, il est connu que $\mathbb{F}_{p}\left(\left(T^{-1}\right)\right)$ est le completé de $\mathbb{F}_{p}(T)$ pour cette valeur absolue.

Dans la suite, $r$ est une puissance de $p, r=p^{t}$, avec $t \geq 1$ entier. Le morphisme de Frobenius défini dans $\mathbb{F}_{p}\left(\left(T^{-1}\right)\right)$ est noté $\alpha \mapsto \alpha^{r}$. Une série formelle, $\alpha \in \mathbb{F}_{p}\left(\left(T^{-1}\right)\right)$, est dite hyperquadratique si l'on a $\alpha=f\left(\alpha^{r}\right)$ où $f$ est une homographie à coefficients dans $\mathbb{F}_{p}[T]$.

Tout élément $\alpha \in \mathbb{F}_{p}\left(\left(T^{-1}\right)\right)$ a un développement en fraction continue (infini si $\alpha$ n'est pas une fraction rationnelle) que l'on notera

$$
\alpha=\left[a_{0}, a_{1}, a_{2}, \ldots, a_{n}, \alpha_{n+1}\right]
$$


où les $a_{i} \in \mathbb{F}_{p}[T]$ (avec $\operatorname{deg}\left(a_{i}\right)>0$ pour $i>0$ ) sont appelés les quotients partiels et les $\alpha_{i} \in \mathbb{F}_{p}\left(\left(T^{-1}\right)\right)$ sont les quotients complets.

\section{Méthode employée et exemple de MahleR}

Dans cette partie nous présentons le raisonnement sur lequel les preuves reposent. Nous commençons par un lemme élémentaire concernant les fractions continues. Une courte démonstration en est donnée dans l'article [3].

Nous rappelons déjà la notation suivante. Soit $P / Q \in \mathbb{F}_{p}(T)$ tel que $P / Q:=\left[a_{1}, a_{2}, \ldots, a_{n}\right]$. Pour tout $x \in \mathbb{F}_{p}(T)$, nous noterons

$$
\left[\left[a_{1}, a_{2}, \ldots, a_{n}\right], x\right]:=\frac{P}{Q}+\frac{1}{x} .
$$

Lemme 1. Soient $a_{1}, \ldots, a_{n}, x \in \mathbb{F}_{p}(T)$. On a la relation suivante :

$$
\left[\left[a_{1}, a_{2}, \ldots, a_{n}\right], x\right]=\left[a_{1}, a_{2}, \ldots, a_{n}, x^{\prime}\right]
$$

avec

$$
x^{\prime}=f_{n} x+g_{n},
$$

où les $f_{n}, g_{n}$ sont des éléments de $\mathbb{F}_{p}\left(a_{1}, a_{2}, \ldots, a_{n}\right)$ (voir [3], page 330).

A l'exception du paragraphe 2.2, nous utilisons ce lemme uniquement dans les cas $n=2$ et $n=3$, qui s'énoncent comme suit.

Lemme 2. Soient $a_{1}, a_{2}, a_{3}, x \in \mathbb{F}_{p}(T)$. On a les relations suivantes :

$$
\begin{gathered}
{\left[\left[a_{1}, a_{2}\right], x\right]=\left[a_{1}, a_{2}, y\right] \text { ò̀ } y=-a_{2}^{-2} x-a_{2}^{-1},} \\
{\left[\left[a_{1}, a_{2}, a_{3}\right], x\right]=\left[a_{1}, a_{2}, a_{3}, y\right] \text {, où } y=\left(a_{2} a_{3}+1\right)^{-2} x-a_{2}\left(a_{2} a_{3}+1\right)^{-1} .}
\end{gathered}
$$

2.1. Premier exemple. Nous allons à présent décrire la suite de quotients partiels d'un ensemble de séries formelles vérifiant un certain type d'équation. Comme corollaire, nous obtenons le développement en fraction continue de la série de Mahler :

$$
\Theta_{r}=1 / T+1 / T^{r}+1 / T^{r^{2}}+\cdots+1 / T^{r^{k}}+\cdots \in \mathbb{F}_{p}\left(\left(T^{-1}\right)\right) .
$$

On peut remarquer que $\Theta_{r}$ est une série algébrique de degré $r$ vérifiant l'équation

$$
T z^{r}-T z+1=0 .
$$

Il convient de noter que le développement en fraction continue de $\Theta_{r}$, donné dans le corollaire 1 est déjà connu, même s'il n'a jamais été présenté sous cette forme. En effet, il est exposé dans [2] (p. 215) et peut aussi être déduit de travaux plus anciens de Shallit sur les fractions continues de certains nombres réels [7].

Théorème 1. Soit $p$ un nombre premier et $r=p^{t}, t \geq 1$, avec $r>2$. Soit $\ell \in \mathbb{N}, \ell \geq 1$ et soit $\left(a_{1}, a_{2}, \ldots, a_{l}\right)$ un $\ell$-uplet de polynômes dans $F_{p}[T]$, avec $a_{i}(T) \in T \mathbb{F}_{p}[T]$, pour tout $i$ impair, $1 \leq i \leq l$. Si $z$ est la fraction continue $z=\left[a_{1}, a_{2}, \ldots, a_{\ell}, z_{\ell+1}\right]$ vérifiant l'équation:

$$
z^{r}=-T^{2} z_{\ell+1}-T
$$

alors la suite de quotients partiels de $z,\left(a_{n}\right)_{n \geq l+1} \in\left(\mathbb{F}_{p}[T]\right)^{\mathbb{N}}$ est définie pour $k \geq 0$ par :

$$
\begin{array}{lll}
a_{\ell+4 k+1}=-\frac{a_{2 k+1}^{r}}{T^{2}}, & a_{\ell+4 k+2}=-T, \\
a_{\ell+4 k+3}=a_{2 k+2}^{r}, & a_{\ell+4 k+4}=T .
\end{array}
$$

Remarque 1. L'existence de la fraction continue vérifiant (44) découle du théorème 1 de l'article [3]. 
Revenons maintenant à la série $\Theta_{r}$. On pose $y:=1 / \Theta_{r}$ et $y:=\left[a_{1}, a_{2}, \ldots, a_{n}, \ldots\right]$. D'après (2), pour $r>2$, on a

$$
\left|T-\frac{1}{\Theta_{r}}\right|=\frac{1}{\left|T^{r-1} \Theta_{r}\right|}=\frac{1}{|T|^{r-2}}<1,
$$

et par conséquent $y=T+1 / y_{2}=\left[T, y_{2}\right]$. D'après (3) on a

$$
\frac{T}{y^{r}}=\frac{T}{y}-1=-\frac{1}{T y_{2}+1}
$$

et donc $y_{2}$ vérifie la relation :

$$
y^{r}=-T^{2} y_{2}-T
$$

En remarquant que l'équation (5) est un cas particulier de l'équation (44), où $\ell=1$ at $a_{1}=T$, nous en déduisons directement le corollaire suivant.

Corollaire 1. On a $\Theta_{r}=\left[0, a_{1}, a_{2}, \ldots, a_{n}, \ldots\right]$ où la suite $\left(a_{i}\right)_{i \geq 1}$ est définie par récurrence, pour $k \geq 0$, par :

$$
\begin{array}{ccccc}
a_{4 k+1}=T, & a_{4 k+2}= & -a_{2 k+1}^{r} / T^{2}, \\
a_{4 k+3}=-T, & a_{4 k+4}= & a_{2 k+2}^{r} .
\end{array}
$$

Démonstration du théorème 1. Nous partons de la relation :

$$
z^{r}=-T^{2} z_{\ell+1}-T,
$$

qui peut être écrite aussi sous la forme $\left[a_{1}^{r}, z_{2}^{r}\right]=-T^{2} z_{\ell+1}-T$, ou encore :

$$
\left[\left[-\frac{a_{1}^{r}}{T^{2}},-T\right],-T^{2} z_{2}^{r}\right]=z_{\ell+1} .
$$

En utilisant le lemme 2 et en tenant en compte du fait que $a_{1}$ est divisible par $T$, nous en déduisons les relations suivantes :

$$
z_{\ell+1}=\left[-\frac{a_{1}^{r}}{T^{2}},-T, z^{\prime}\right], \text { avec } z^{\prime}=z_{2}^{r}+T^{-1} .
$$

Puisque $\left|z^{\prime}\right|>1$, on en déduit que $z^{\prime}=z_{l+3}$. On a donc

$$
a_{\ell+1}=-\frac{a_{1}^{r}}{T^{2}}, a_{\ell+2}=-T \text { et } z_{l+3}=z_{2}^{r}+\frac{1}{T} .
$$

Nous appliquons de nouveau le même raisonnement et nous obtenons :

$$
z_{l+3}=z_{2}^{r}+\frac{1}{T}=\left[a_{2}^{r}+\frac{1}{T}, z_{3}^{r}\right]=\left[a_{2}^{r}, T, z^{\prime \prime}\right]
$$

avec $z^{\prime \prime}=-T^{-2} z_{3}^{r}-T^{-1}$. Puisque $r>2$, on a $\left|z^{\prime \prime}\right|>1$ et alors, par identification, $z^{\prime \prime}=z_{l+5}$. On a donc

$$
a_{\ell+3}=a_{2}^{r}, a_{\ell+4}=T \text { et } z_{l+5}=-T^{-2} z_{3}^{r}-T^{-1} .
$$

En résumé,

$$
z_{\ell+1}=\left[-\frac{a_{1}^{r}}{T^{2}},-T, a_{2}^{r}, T, z_{\ell+5}\right] .
$$

Plus généralement, par une simple récurrence sur $k$, on obtient que :

$$
z_{2 k+1}^{r}=-T^{2} z_{\ell+4 k+1}-T .
$$

Puisque $a_{2 k+1} \in T \mathbb{F}_{p}[T]$, nous obtenons comme précédemment :

$$
z_{\ell+4 k+1}=\left[-\frac{a_{2 k+1}^{r}}{T^{2}},-T, a_{2 k+2}^{r}, T, z_{\ell+4 k+5}\right],
$$

ce qui termine la démonstration. 
2.2. Le contexte général. Dans cette partie nous énonçons le raisonnement général et les notations utilisées dans les preuves qui suivent. Ceux-ci restent proches de ceux utilisés par Mills et Robbins dans [5].

Soit $(P, Q, R) \in \mathbb{F}_{p}[T]^{3}$ et soient $m, n$ deux entiers strictement positifs, $m<n$. On dit que $z$ satisfait une relation du type $(P, Q, R, m, n)$ si on a :

$$
P z_{m}^{r}=Q z_{n}+R .
$$

Dans la suite, nous considérons une série $z=\left[a_{1}, a_{2}, \ldots\right] \in \mathbb{F}_{p}\left(\left(T^{-1}\right)\right)$ satisfaisant (6) , avec un triplet $(P, Q, R)$ bien choisi. En fait, il est probable que cette relation soit vraie pour presque toutes les séries formelles hyperquadratiques, mais à notre connaissance, aucun résultat général ne le confirme (le lecteur peut consulter [3], page 333, pour quelques commentaires à ce sujet).

On suppose connus les $n-1$ premiers coefficients partiels : $a_{1}, a_{2}, \ldots, a_{n-1}$ (qui peuvent être vus comme une "donnée de départ"). La relation (6) implique :

$$
P a_{m}^{r}+\frac{P}{z_{m+1}^{r}}=Q z_{n}+R,
$$

car, par définition, $z_{m}=\left[a_{m}, z_{m+1}\right]$. Ainsi, nous obtenons :

$$
\frac{P a_{m}^{r}-R}{Q}+\frac{P}{Q z_{m+1}^{r}}=z_{n} .
$$

Puisque

$$
\frac{P a_{m}^{r}-R}{Q} \in \mathbb{F}_{p}(T),
$$

cette expression a un développement en fraction continue qui est fini. Il existe ainsi des polynômes $\lambda_{1}, \lambda_{2}, \ldots, \lambda_{\ell} \in \mathbb{F}_{p}[T]$, que l'on peut calculer, tels que :

$$
\frac{P a_{m}^{r}-R}{Q}=\left[\lambda_{1}, \lambda_{2}, \ldots, \lambda_{\ell}\right] .
$$

La relation (7) devient :

$$
\left[\left[\lambda_{1}, \lambda_{2}, \ldots, \lambda_{\ell}\right], \frac{Q z_{m+1}^{r}}{P}\right]=z_{n},
$$

et le lemme 1 implique alors que :

$$
z_{n}=\left[\lambda_{1}, \lambda_{2}, \ldots, \lambda_{\ell}, z^{\prime}\right] .
$$

A ce moment, si $\left|z^{\prime}\right|>1$, nous pouvons déjà identifier $a_{n}=\lambda_{1}, a_{n+1}=\lambda_{2}, \ldots, a_{n+l-1}=\lambda_{l}$ et $z_{n+l}=z^{\prime}$.

Par le lemme 1 on déduit donc :

$$
z_{n+\ell}=f_{\ell} \frac{Q z_{m+1}^{r}}{P}+g_{\ell},
$$

où $f_{\ell}$ et $g_{\ell}$ appartiennent à $\mathbb{F}_{p}(T)$ (voir la formule (1) ). Il existe donc trois polynômes $P_{1}, Q_{1}, R_{1} \in$ $\mathbb{F}_{p}[T]$, qu'on peut déterminer explicitement, tels que :

$$
P_{1} z_{m+1}^{r}=Q_{1} z_{n+\ell}+R_{1} .
$$

En résumé, la connaissance de $P, Q, R$ et $a_{m}$ nous permet de déterminer les $\ell$ nouveaux quotients partiels : $a_{n}, a_{n+1}, \ldots, a_{n+\ell-1}$ et une nouvelle équation :

$$
P_{1} z_{m+1}^{r}=Q_{1} z_{n+\ell}+R_{1} .
$$

Dans la suite, nous noterons ce raisonnement par :

$$
\left(P, Q, R, m, n: a_{m}\right) \rightarrow\left(P_{1}, Q_{1}, R_{1}, m+1, n+\ell: a_{n}, a_{n+1}, \ldots, a_{n+\ell-1}\right) .
$$

Plus généralement, si $X:=(P, Q, R, m, n)$ et $Y:=\left(P_{\ell}, Q_{\ell}, R_{\ell}, m+\ell, n+k\right)$, nous noterons :

$$
\left(X: a_{m}, a_{m+1}, \ldots, a_{m+\ell-1}\right) \rightarrow\left(Y: a_{n}, a_{n+1}, \ldots, a_{n+k-1}\right)
$$


pour désigner la suite des raisonnements suivants :

$$
\begin{cases}\left(X: a_{m}\right) & \rightarrow\left(X_{1}: a_{n}, a_{n+1}, \ldots, a_{n+k_{1}-1}\right) \\ \left(X_{1}: a_{m+1}\right) & \rightarrow\left(X_{2}: a_{n+k_{1}}, a_{n+k_{1}+1}, \ldots a_{n+k_{2}-1}\right) \\ & \vdots \\ \left(X_{\ell-1}: a_{m+\ell-1}\right) & \rightarrow\left(Y: a_{n+k_{\ell-1}}, a_{n+k_{\ell-1}+1}, \ldots, a_{n+k-1}\right) .\end{cases}
$$

où $X_{i}:=\left(P_{i}, Q_{i}, R_{i}, m+i, n+k_{i}\right)$, pour $1 \leq i \leq \ell-1$. La nouvelle équation va donc relier $z_{m+\ell}$ et $z_{n+k}$ de la manière suivante :

$$
P_{\ell} z_{m+\ell}^{r}=Q_{\ell} z_{n+k}+R_{\ell} .
$$

Nous remarquons que, lorsque $l=n-m$, la relation (8) s'écrit :

$$
\left(P, Q, R, m, n: a_{m}, a_{m+1}, \ldots, a_{n-1}\right) \rightarrow\left(P_{n-m}, Q_{n-m}, R_{n-m}: a_{n}, a_{n+1} \ldots, a_{n+k-1}\right),
$$

ce qui signifie que la connaissance de $a_{m}, a_{m+1}, \ldots, a_{n-1}$ nous permet de calculer les $k$ quotients partiels suivants, à savoir $a_{n}, a_{n+1}, \ldots, a_{n+k-1}$. Nous pouvons alors appliquer de nouveau le même raisonnement, à une équation du type $\left(P_{n-m}, Q_{n-m}, R_{n-m}, m+n-1, n+k\right)$ ayant comme "donnée de départ" les quotients partiels $a_{n}, a_{n+1}, \ldots, a_{n+k-1}$. L'itération de ce procédé permet ainsi d'obtenir tous les quotients partiels de la série $z$.

Soient $W, W^{\prime}$ deux suites finies de polynômes à coefficients dans $\mathbb{F}_{p}$. On note $|W|$ la longueur de $W$, c'est-à-dire le nombre de ses termes. Alors la relation (8) peut être écrite aussi :

$$
(P, Q, R, m, n: W) \rightarrow\left(P_{|W|}, Q_{|W|}, R_{|W|}, m+|W|, n+\left|W^{\prime}\right|: W^{\prime}\right) .
$$

Pour alléger les notations, nous nous permettons parfois d'écrire :

$$
(P, Q, R, m, n: W) \rightarrow\left(P_{|W|}, Q_{|W|}, R_{|W|}: W^{\prime}\right) .
$$

\section{Généralisation de la cubique de Baum et Sweet}

Avant d'énoncer notre résultat principal, nous allons tout d'abord introduire quelques définitions.

Définition 1. On définit une suite de polynômes à coefficients dans $\mathbb{F}_{p},\left(\Gamma_{k}\right)_{k \geq 1}$, de la façon suivante. On pose $\Gamma_{1}:=-T, T^{r}, T$. Pour $k>1, \Gamma_{k}$ est défini récursivement par :

$$
\Gamma_{k}:=a_{1}, a_{2}, \ldots, a_{2^{k+1}-1} \text { et } \Gamma_{k+1}:=b_{1}, b_{2}, \ldots, b_{2^{k+2}-1},
$$

où

$$
b_{2 i-1}=(-1)^{i+k} T \quad \text { pour } 1 \leq i \leq 2^{k+1}
$$

et

$$
b_{4 i}=a_{2 i}^{r} / T^{2} \text { pour } 1 \leq i \leq 2^{k}-1, \quad b_{4 i-2}=-a_{2 i-1}^{r} \text { pour } 1 \leq i \leq 2^{k} .
$$

Définition 2. On définit la suite des polynômes à coefficients dans $\mathbb{F}_{p},\left(\Lambda_{k}\right)_{k \geq 1}$, de la façon suivante. On pose $\Lambda_{1}:=T+1, T-1, \Lambda_{2}:=T,-T^{r}+1,-T$. Pour $k \geq 3, \Lambda_{k}$ est défini récursivement par :

$$
\Lambda_{k}:=\Lambda_{k-2},-T^{\lambda_{k-1}}, \Gamma_{k-2},
$$

où $\left(\lambda_{k}\right)_{k \geq 1}$ est défini comme suit :

$$
\lambda_{1}=r, \quad \lambda_{k+1}=r \lambda_{k}-2 .
$$

Etant donnée une suite $W=a_{1}, \ldots, a_{m}$ à valeurs dans $\mathbb{F}_{p}[T]$, on note $-W:=-a_{1}, \ldots,-a_{m}$ et $\bar{W}:=a_{m}, a_{m-1}, \ldots, a_{1}$.

Définition 3. On définit la suite des polynômes à coefficients dans $\mathbb{F}_{p},\left(\Omega_{k}\right)_{k \geq 1}$, de la façon suivante. On pose $\Omega_{1}:=-T^{\omega_{1}}$. Pour $k \geq 2, \Omega_{k}$ est défini récursivement par :

$$
\Omega_{k}:=\Omega_{k-1}, \Lambda_{k-1},-T^{\omega_{k}},-\bar{\Lambda}_{k-1},-\bar{\Omega}_{k-1},
$$

où $\left(\omega_{k}\right)_{k \geq 1}$ est défini comme suit :

$$
\omega_{1}=r-2, \quad \omega_{k+1}=r \omega_{k}-2 .
$$

Nous notons $\Omega_{\infty}$ la suite infinie commençant par $\Omega_{k}$, pour tout $k \geq 1$. 
Avec les notations ci-dessus, nous présentons maintenant notre résultat principal.

Théorème 2. Soit $p$ un nombre premier et $r=p^{t}$, où $t \geq 1$, avec $r>2$. L'équation

$$
T X^{r+1}+X-T=0
$$

a une unique racine dans le corps $\mathbb{F}_{p}\left(\left(T^{-1}\right)\right)$ dont le développement en fraction continue est :

$$
\left[1,-T-1, \Omega_{\infty}\right] \text {. }
$$

3.1. Démonstration du théorème 2. Tout d'abord, nous montrons que l'équation :

$$
T X^{r+1}+X-T=0
$$

a une unique solution dans le corps $\mathbb{F}_{p}\left(\left(T^{-1}\right)\right)$.

En effet, de (10), on déduit que :

$$
X=\frac{T}{T X^{r}+1} .
$$

Nous considérons maintenant l'application $f(X)=T /\left(T X^{r}+1\right)$ définie sur $\mathbb{F}_{p}\left(\left(T^{-1}\right)\right)$ à valeurs dans $\mathbb{F}_{p}\left(\left(T^{-1}\right)\right)$. Il n'est pas difficile de voir que $f$ est une application strictement contractante (puisque $r>2$ ) et nous savons que $\mathbb{F}_{p}\left(\left(T^{-1}\right)\right.$ ) est un espace complet pour la distance ultramétrique usuelle. Ainsi, par utilisation du théorème du point fixe, l'équation $f(X)=X$ a une unique solution dans $\mathbb{F}_{p}\left(\left(T^{-1}\right)\right)$. Celle-ci est donc l'unique solution de l'équation (10), que nous noterons dans la suite $B S_{r}$.

Soit $z$ la série définie par :

$$
B S_{r}=1+\frac{1}{(-T-1)+1 / z}
$$

D'après (11) et (12) on a :

$$
z=\frac{\left(-T^{r}+T-1\right) z^{r}+1}{T^{2} z^{r}}
$$

ce qui entraîne

$$
\left|z-\frac{-T^{r}+T-1}{T^{2}}\right|=\frac{1}{\left|T^{2} z^{r}\right|}<\frac{1}{\left|T^{4}\right|} .
$$

Autrement dit $\left(-T^{r}+T-1\right) / T^{2}$ est une réduite de $z$, donc les 3 premiers quotients partiels de $z$ sont $-T^{r-2}, T+1$ et $T-1$. Ainsi $z=\left[-T^{r-2}, T+1, T-1, z_{4}\right]$ et d'après (13) on obtient la relation suivante :

$$
z^{r}=T^{2} z_{4}+(T+1)
$$

Le théorème 2 est alors une conséquence directe de proposition ci-dessous.

Proposition 1. Soit $z$ la fraction continue infinie $z:=\left[-T^{r-2}, T+1, T-1, z_{4}\right]$ satisfaisant :

$$
z^{r}=T^{2} z_{4}+(T+1)
$$

Alors la suite de quotients partiels de $z$ est $\Omega_{\infty}$.

Notation 1. On dit qu'une équation est du type $A_{1}$ si $P=1, Q=T^{2}$ et $R=T+1$ et on note $A_{1}:=\left(1, T^{2}, T+1\right)$. De la même manière, nous allons définir les équations des types suivants :

$$
\begin{aligned}
& A_{2}:=\left(1, T^{2},-T+1\right), \\
& A_{3}:=(T,-T,-1), \\
& A_{4}:=\left(1, T^{2},-T\right), \\
& A_{5}:=(T,-T, 1), \\
& A_{6}:=\left(1, T^{2}, T\right) .
\end{aligned}
$$

Dans ce qui suit, nous utiliserons les notations décrites dans la partie 2.2 . 
Lemme 3. Soient $m, n \in \mathbb{N}$ tels que $m<n$ et soit $a \in \mathbb{F}_{p}[T]$. On a les relations suivantes :

$$
\begin{aligned}
& \left(A_{1}, m, n: a\right) \rightarrow\left(A_{2}, m+1, n+3: \frac{a^{r}}{T^{2}},-T+1,-T-1\right) \text { si } a \equiv 0[T], \\
& \left(A_{1}, m, n: a\right) \rightarrow\left(A_{5}, m+1, n+2: \frac{(a-1)^{r}}{T^{2}},-T\right) \text { si } a \equiv 1[T], \\
& \left(A_{2}, m, n: a\right) \rightarrow\left(A_{1}, m+1, n+3: \frac{a^{r}}{T^{2}}, T+1, T-1\right) \text { si } a \equiv 0[T], \\
& \left(A_{2}, m, n: a\right) \rightarrow\left(A_{3}, m+1, n+2: \frac{(a-1)^{r}}{T^{2}}, T\right) \text { si } a \equiv 1[T], \\
& \left(A_{3}, m, n: a\right) \rightarrow\left(A_{4}, m+1, n+2:-a^{r},-T\right) \text { pour tout } a \in \mathbb{F}_{p}[T], \\
& \left(A_{4}, m, n: a\right) \rightarrow\left(A_{3}, m+1, n+2: \frac{a^{r}}{T^{2}}, T\right) \text { si } a \equiv 0[T], \\
& \left(A_{4}, m, n: a\right) \rightarrow\left(A_{2}, m+1, n+3: \frac{(a+1)^{r}}{T^{2}}, T+1, T-1\right) \text { si } a \equiv-1[T], \\
& \left(A_{5}, m, n: a\right) \rightarrow\left(A_{6}, m+1, n+2:-a^{r}, T\right) \text { pour tout } a \in \mathbb{F}_{p}[T], \\
& \left(A_{6}, m, n: a\right) \rightarrow\left(A_{5}, m+1, n+2: \frac{a^{r}}{T^{2}},-T\right) \text { si } a \equiv 0[T], \\
& \left(A_{6}, m, n: a\right) \rightarrow\left(A_{2}, m+1, n+3: \frac{(a+1)^{r}}{T^{2}},-T+1,-T-1:\right) \text { si } a \equiv-1[T] .
\end{aligned}
$$

Démonstration. Nous allons prouver le premier cas, c'est à dire le cas où $z$ satisfait une relation du type $A_{1}$, avec $a \equiv 0[T]$ :

$$
z_{m}^{r}=T^{2} z_{n}+(T+1) .
$$

Cette relation s'écrit aussi sous la forme : $\left[a^{r}, z_{m+1}^{r}\right]=T^{2} z_{n}+(T+1)$ ou bien

$$
\frac{a^{r}}{T^{2}}-\frac{T+1}{T^{2}}+\frac{1}{T^{2} z_{m+1}}=z_{n} .
$$

Puisque $a$ est divisible par $T$, nous obtenons :

$$
\left[\left[\frac{a^{r}}{T^{2}},-T+1,-T-1\right], T^{2} z_{m+1}\right]=z_{n} .
$$

En appliquant le lemme 2, on en déduit que

$$
z_{n}=\left[\frac{a^{r}}{T^{2}},-T+1,-T-1, z_{n+3}\right]
$$

et

$$
z_{m+1}^{r}=T^{2} z_{n+3}+(-T+1) .
$$

Ainsi nous obtenons les nouveaux quotients partiels $a_{n}=\frac{a^{r}}{T^{2}}, a_{n+1}=-T+1, a_{n+2}=-T-1$ et une équation du type $A_{2}:=\left(1, T^{2},-T+1\right)$.

Les autres cas se déduisent de manière analogue, en appliquant le raisonnement précédent et, en particulier, le lemme 2 ,

Notation 2. Soient $i, j \in \mathbb{N}^{*}, i, j \leq m$ et $W=a_{1}, a_{2}, \ldots, a_{m}$. On notera

$$
{ }^{(i)} W:=a_{i+1}, a_{i+2}, \ldots, a_{m} \text { et } W^{(j)}:=a_{1}, a_{2}, \ldots, a_{m-j} .
$$

Lorsque $i+j<m-1$, on notera

$$
{ }^{(i)} W^{(j)}:=a_{i+1}, \ldots, a_{m-j} .
$$

La proposition 1 est une conséquence immédiate de la proposition 2. 
Proposition 2. Pour tout $k \in \mathbb{N}^{*}$, on a la relation suivante :

$$
\left(A_{1}, 1,4: \Omega_{k}\right) \rightarrow\left(A_{2}, 1+\left|\Omega_{k}\right|,\left|\Omega_{k+1}\right|:{ }^{(3)} \Omega_{k+1}^{(1)}\right) .
$$

Remarque 2. Soit $k \in \mathbb{N}$. Nous notons $\ell_{k}$ la longueur du mot fini $\Omega_{k}$. La proposition précédente peut être traduite de la manière suivante. On suppose connus les $\ell_{k}$ premiers quotients de $z$. Alors, si on applique $\ell_{k}$ fois le procédé énoncé dans le paragraphe 2.2 à l'équation (14) nous obtenons :

et la nouvelle relation sera :

$$
z_{4}=\left[{ }^{(3)} \Omega_{k+1}^{(1)}, z_{\ell_{k+1}}\right]
$$

$$
z_{\ell_{k}+1}=T^{2} z_{\ell_{k+1}}+(T+1)
$$

Pour démontrer la proposition 2, nous allons utiliser les lemmes suivants.

Lemme 4. Soient $k, m, n$ des entiers strictement positifs, $m<n$. On a les relations suivantes: - si $k$ est pair, alors :

$$
\begin{aligned}
& \left(A_{5}, m, n: \Gamma_{k}\right) \rightarrow\left(A_{6}:{ }^{(1)} \Gamma_{k+1}\right), \\
& \left(A_{5}, m, n:-\bar{\Gamma}_{k}\right) \rightarrow\left(A_{6}:{ }^{(1)}\left(-\bar{\Gamma}_{k+1}\right)\right) ;
\end{aligned}
$$

- si $k$ est impair, alors :

$$
\begin{aligned}
& \left(A_{3}, m, n: \Gamma_{k}\right) \rightarrow\left(A_{4}:{ }^{(1)} \Gamma_{k+1}\right), \\
& \left(A_{3}, m, n:-\bar{\Gamma}_{k}\right) \rightarrow\left(A_{4}:{ }^{(1)}\left(-\bar{\Gamma}_{k+1}\right)\right) .
\end{aligned}
$$

Démonstration. Tout d'abord, on remarque que, pour tout $k \in \mathbb{N}$, les termes de $\Gamma_{k}$ sont des polynômes divisibles par $T$.

Soient $a, b \in T \mathbb{F}_{p}(T)$. Alors, à l'aide du lemme 3 on peut déduire :

$$
\left(A_{5}, m, n: a, b\right) \rightarrow\left(A_{5}, m+2, n+4:-a^{r}, T, b^{r} / T^{2}, T\right) .
$$

Plus généralement, si $a_{1}, a_{2}, \ldots, a_{2 i} \in T \mathbb{F}_{p}[T]$ alors :

$$
\left(A_{5}, m, n: a_{1}, a_{2} \ldots, a_{2 i}\right) \rightarrow\left(A_{5}:-a_{1}^{r}, T, a_{2}^{r} / T^{2}, T, \ldots,-a_{2 i-1}^{r}, T, a_{2 i}^{r} / T^{2}, T\right) .
$$

Soit $k \in \mathbb{N}$ et $\Gamma_{k}:=a_{1}, a_{2}, \ldots, a_{2^{k+1}-1}$. La suite $\Gamma_{k}$ a un nombre impair d'éléments; ainsi, nous devons appliquer le lemme 3 à son dernier terme aussi. On obtient :

$$
\left(A_{5}, m, n: \Gamma_{k}\right) \rightarrow\left(A_{6}:-a_{1}^{r}, T, a_{2}^{r} / T^{2}, T, \ldots,-a_{2 i-1}^{r}, T, a_{2 i}^{r} / T^{2}, T,-a_{2 i+1}^{r}, T\right) .
$$

Dans le cas où $k$ est pair, le premier terme de $\Gamma_{k+1}$ est $b_{1}:=-T$. Donc, par définition,

$$
{ }^{(1)} \Gamma_{k+1}=b_{2}, \ldots, b_{2^{k+2}-1}=-a_{1}^{r}, T, \ldots,-a_{2 i-1}^{r}, T, a_{2 i}^{r} / T^{2}, T,-a_{2 i+1}^{r},
$$

ce qui coïncide avec notre résultat.

Les autres cas se démontrent de manière analogue.

Lemme 5. Soient $k, m, n$ des entiers strictement positifs, $m<n$. On a les relations suivantes : - si $k$ est pair, alors :

$$
\begin{aligned}
& \left(A_{2}, m, n: \Lambda_{k}\right) \rightarrow\left(A_{6}: T^{r-2}, \Lambda_{k+1}\right), \\
& \left(A_{5}, m, n:-\bar{\Lambda}_{k}\right) \rightarrow\left(A_{1}:{ }^{(1)}\left(-\bar{\Lambda}_{k+1}\right), T^{r-2}, T+1, T-1\right) .
\end{aligned}
$$

- si $k$ est impair, alors :

$$
\begin{aligned}
& \left(A_{2}, m, n: \Lambda_{k}\right) \rightarrow\left(A_{4}: T^{r-2}, \Lambda_{k+1}\right), \\
& \left(A_{3}, m, n:-\bar{\Lambda}_{k}\right) \rightarrow\left(A_{1}:{ }^{(1)}\left(-\bar{\Lambda}_{k+1}\right), T^{r-2}, T+1, T-1\right) .
\end{aligned}
$$

Démonstration. Nous allons démontrer ici la relation (15). Les relations (16), (17) et (18) peuvent être démontrées de manière analogue. On raisonne par récurrence sur $k$.

Nous commençons par le cas où $k=2$. Par définition :

$$
\Lambda_{2}=T,-T^{r}+1,-T
$$


et

$$
\Lambda_{3}=T+1, T-1,-T^{r^{2}-2},-T, T^{r}, T .
$$

En appliquant les formules du lemme 3, on a :

$$
\begin{aligned}
\left(A_{2}, m, n: T\right) & \rightarrow\left(A_{1}, m+1, n+3: T^{r-2}, T+1, T-1\right) \\
\left(A_{1}, m+1, n+3:-T^{r}+1\right) & \rightarrow\left(A_{5}, m+2, n+5:-T^{r^{2}-2},-T\right) \\
\left(A_{5}, m+2, n+5:-T\right) & \rightarrow\left(A_{6}:-T^{r}, T\right)
\end{aligned}
$$

Par conséquent, (15) est prouvé pour $k=2$.

Nous supposons à présent que $k$ est un entier pair, $k>2$, et que la relation (15) est vraie pour $k-2$. Nous allons la montrer maintenant pour $k$.

Par définition,

Par l'hypothèse de récurrence,

$$
\Lambda_{k}=\Lambda_{k-2},-T^{\lambda_{k-1}}, \Gamma_{k-2}
$$

$$
\left(A_{2}, m, n: \Lambda_{k-2}\right) \rightarrow\left(A_{6}, m+\left|\Lambda_{k-2}\right|, n+\left|\Lambda_{k-1}\right|+1: T^{r-2}, \Lambda_{k-1}\right) .
$$

En appliquant les lemmes 3 et 4 nous avons aussi :

$$
\begin{aligned}
\left(A_{6}, m+\left|\Lambda_{k-2}\right|, n+\left|\Lambda_{k-1}\right|+1:-T^{\lambda_{k-1}}\right) & \rightarrow\left(A_{5}:-T^{\lambda_{k}},-T\right) \\
\left(A_{5}, m+\left|\Lambda_{k-2}\right|+1, n+\left|\Lambda_{k-1}\right|+3: \Gamma_{k-2}\right) & \rightarrow\left(A_{6}:{ }^{(1)} \Gamma_{k-1}\right) .
\end{aligned}
$$

En réunissant tous ces relations et en tenant en compte que, pour tous les $k$ pairs, $\Gamma_{k-1}$ commence par $-T$, on obtient le résultat.

Démonstration de la proposition 圆. Nous allons prouver par récurrence sur $k$ que, pour tous $m, n$ tels que $m<n$, on a les relations suivantes :

$$
\begin{gathered}
\left(A_{1}, m, n: \Omega_{k}\right) \rightarrow\left(A_{2}:{ }^{(3)} \Omega_{k+1}^{(1)}\right) \\
\left(A_{1}, m, n:-\bar{\Omega}_{k}\right) \rightarrow\left(A_{2}:{ }^{(3)}\left(-\bar{\Omega}_{k+1}\right)^{(1)}\right) .
\end{gathered}
$$

Lorsque $m=1, n=4$, la relation (19) implique la proposition 2. On commence par le cas où $k=1$. On a :

$$
\begin{aligned}
\Omega_{1} & =-T^{r-2},-\overline{\Omega_{1}}=T^{r-2}, \\
\Omega_{2} & =-T^{r-2}, T+1, T-1,-T^{\omega_{2}},-T+1,-T-1, T^{r-2}, \\
-\bar{\Omega}_{2} & =-T^{r-2}, T+1, T-1, T^{\omega_{2}},-T+1,-T-1, T^{r-2} .
\end{aligned}
$$

D'autre part, le lemme 3 implique que pour tous $m, n, m<n$ :

$$
\left(A_{1}, m, n: \pm T^{r-2}\right) \rightarrow\left(A_{2}: \pm T^{r(r-2)-2},-T+1,-T-1\right),
$$

et puisque $\omega_{2}=r(r-2)-2$ les relations (19) et (20) sont établies pour $k=1$.

Soit $k>1$. Nous supposons maintenant que (19) et (20) sont vraies pour $k-1$ et tous $m, n$, $m<n$. A cause du lemme 5, nous devons distinguer deux cas : le cas où $k$ est pair et le cas où $k$ est impair. Nous allons supposer maintenant que $k$ est pair.

Fixons $m, n \in \mathbb{N}, m<n$.

D'après (9) on peut écrire les deux relations suivantes :

$$
\begin{aligned}
\Omega_{k} & =\Omega_{k-1}, \Lambda_{k-1},-T^{\omega_{k}},-\bar{\Lambda}_{k-1},-\bar{\Omega}_{k-1}, \\
-\bar{\Omega}_{k} & =\Omega_{k-1}, \Lambda_{k-1}, T^{\omega_{k}},-\bar{\Lambda}_{k-1},-\bar{\Omega}_{k-1},
\end{aligned}
$$

afin d'appliquer notre algorithme à chaque sous-suite qui apparaît dans les expressions de $\Omega_{k}$ et $-\bar{\Omega}_{k}$.

Soient $1 \leq i, i^{\prime} \leq 6$ et $W, W^{\prime}$ des suites finies de polynômes sur $\mathbb{F}_{p}$. Pour alléger l'écriture, nous nous permettons d'utiliser la notation :

$$
\left(A_{i}: W\right) \rightarrow\left(A_{i^{\prime}}: W^{\prime}\right)
$$


les indices $m$ et $n$ étant sous-entendus.

Par l'hypothèse de récurrence, on a :

$$
\begin{aligned}
& \left(A_{1}: \Omega_{k-1}\right) \rightarrow\left(A_{2}:{ }^{(3)} \Omega_{k}^{(1)}\right), \\
& \left(A_{1}:-\bar{\Omega}_{k-1}\right) \rightarrow\left(A_{2}:{ }^{(3)}\left(-\bar{\Omega}_{k}\right)^{(1)}\right) .
\end{aligned}
$$

Les lemmes 5 et 3 impliquent :

$$
\begin{aligned}
& \left(A_{2}: \Lambda_{k-1}\right) \rightarrow\left(A_{4}: T^{r-2}, \Lambda_{k}\right), \\
& \left(A_{4}: \pm T^{\omega_{k}}\right) \rightarrow\left(A_{3}: \pm T^{\omega_{k+1}}, T\right), \\
& \left(A_{3}:-\bar{\Lambda}_{k-1}\right) \rightarrow\left(A_{1}:{ }^{(1)}\left(-\bar{\Lambda}_{k}\right), T^{r-2}, T+1, T-1\right) .
\end{aligned}
$$

En résumé, on a :

$$
\begin{aligned}
& \left(A_{1}: \Omega_{k}\right) \rightarrow\left(A_{2}:{ }^{(3)} \Omega_{k}^{(1)}, T^{r-2}, \Lambda_{k}, T^{\omega_{k+1}}, T,{ }^{(1)}\left(-\bar{\Lambda}_{k}\right), T^{r-2}, T+1, T-1,{ }^{(3)}\left(-\bar{\Omega}_{k}\right)^{(1)}\right), \\
& \left(A_{1}: \bar{\Omega}_{k}\right) \rightarrow\left(A_{2}:{ }^{(3)} \Omega_{k}^{(1)}, T^{r-2}, \Lambda_{k},-T^{\omega_{k+1}}, T,{ }^{(1)}\left(-\bar{\Lambda}_{k}\right), T^{r-2}, T+1, T-1,{ }^{(3)}\left(-\bar{\Omega}_{k}\right)^{(1)}\right) .
\end{aligned}
$$

Nous remarquons que pour tous les $k>1, \Omega_{k}$ commence par $-T^{r-2}, T+1, T-1$ et il se termine par $-T+1,-T-1, T^{r-2}$. De même, $-\bar{\Lambda}_{k}$ commence par $T$ (puisque $\Gamma_{k-2}$ se termine par $-T$ ). En combinant les relations précédentes, nous en déduisons (19) et (20).

Le cas où $k$ est impair se traite de manière analogue.

Nous avons observé que tout le raisonnement ci-dessus pour obtenir le développement en fraction continue de $B S_{r}$ est basé sur le fait que le premier des 3 quotients partiels de départ $\left(-T^{r-2}, T+1, T-1\right)$ est divisible par $T$. Ainsi, nous pouvons obtenir un résultat plus général en remplaçant $-T^{r-2}$ par un polynôme $P$ arbitraire, divisible par $T$. En utilisant les mêmes notations qu'au début de ce paragraphe, nous avons le théorème ci-dessous dont la preuve s'obtient comme précédemment.

Théorème 3. Soit $P \in T \mathbb{F}_{p}[T]$. On définit la suite des polynômes à coefficients dans $\mathbb{F}_{p}$, $\left(\Omega_{k}(P)\right)_{k \geq 0}$, de la façon suivante.

On pose $\Omega_{1}(P):=P$. Pour $k \geq 2, \Omega_{k}(P)$ est défini récursivement par :

$$
\Omega_{k}(P):=\Omega_{k-1}(P), \Lambda_{k-1}, T^{\omega_{k-1}}(P / T)^{r^{k-1}},-\bar{\Lambda}_{k-1},-\bar{\Omega}_{k-1} .
$$

Si $z$ est la fraction continue infinie $z:=\left[P, T+1, T-1, z_{4}\right]$ satisfaisant :

$$
z^{r}=T^{2} z_{4}+(T+1),
$$

alors la suite de quotients partiels de $z$ est $\Omega_{\infty}(P)$, où $\Omega_{\infty}(P)$ est la suite infinie commençant par $\Omega_{k}(P)$, pour tout $k \geq 1$.

Remarque 3. L'existence de la fraction continue $z$ vérifiant (21) découle aussi du théorème 1 de l'article [3]. De plus, il est facile de voir que $z$ satisfait l'équation algèbrique :

$$
T^{2} z^{r+1}=\left(P T^{2}+T-1\right) z^{r}+1 .
$$

\section{REMERCIEMENTS}

Je tiens à remercier tout particulièrement Alain Lasjaunias, qui m'a indiqué ce sujet, pour les nombreuses discussions que nous avons eues et pour ses commentaires fort utiles au cours de mon travail. Je remercie également mon directeur de thèse, Boris Adamczewski, pour ses remarques judicieuses qui m'ont beaucoup aidé dans la rédaction du présent travail. 


\section{RÉFÉRENCES}

[1] L. Baum and M. Sweet, Continued fractions of algebraic power series in characteristic 2, Annals of Mathematics 103 (1976), 593-610.

[2] A.Lasjaunias, A Survey of Diophantine approximation in Fields of Power Series, Monatshefte fur Mathematik 130 (2000), p. 211-229.

[3] A. Lasjaunias, Continued fractions for hyperquadratic power series over a finite field, Finite Fields and their Applications 14 (2008), 329-350.

[4] K. Mahler, On a theorem of Liouville in fields of positive characteristic, Canadian Journal of Mathematics 1 (1949), 397-400.

[5] W. Mills and D. Robbins, Continued fractions for certain algebraic power series, Journal of Number Theory 23 (1986), 388-404.

[6] W. Schmidt, On continued fractions and diophantine approximation in power series fields, Acta Arithmetica 95 (2000), 139-166.

[7] J. Shallit, Simple continued fractions for some irrational numbers, Journal of Number Theory 11 (1979), 209-217.

Université de Lyon, Université Lyon 1, Institut Camille Jordan, UmR 5208 du CNRS, 43, Boulevard du 11 novembre 1918, F-69622 Villeurbanne Cedex, France, firicel@math.univ-lyon1.Fr 